\title{
Scanning Transmission Electron Microscopy Investigation of the Structure of Multilayered Perpendicular Magnetic Tunnel Junctions
}

\author{
Danielle Reifsnyder Hickey ${ }^{1}$ and K. Andre Mkhoyan ${ }^{1}$ \\ ${ }^{1}$ Department of Chemical Engineering and Materials Science, University of Minnesota, \\ Minneapolis, MN 55455, United States
}

The composition and morphology of multilayered magnetic materials has important consequences for magnetic memory applications. CoFeB/MgO interfaces, specifically, exhibit perpendicular magnetic anisotropy, which can be utilized in perpendicular magnetic tunnel junctions (p-MTJs) [1]. Such tunnel junctions are used for read heads, magnetic random access memory, and logic elements [1-3]. The ability to control magnetism using an electric field alone has increased their potential [4], and significant effort has been devoted to fabricating new compositions, characterizing the composition and orientation of the nanostructures that form, and decreasing the layer thickness to enable an electric field to penetrate into the structure despite the screening of electrons [1-4].

Devices based on p-MTJs have great promise, and the realization of predicted performance levels depends on the characteristics of the interfaces, which can vary according to the preparation conditions. Of particular interest is the quality of the interfaces between the various layers. Additionally, MTJ structures often must be compatible with high-temperature processing conditions to improve their performance or be successfully integrated into functional devices [3]. The relative crystallinity and orientation of the layers, the segregation of phases within a layer during annealing, and the migration or diffusion of metals across layers can all affect the performance of devices [3,5]. In certain cases, structural changes during high-temperature treatment can completely destroy the perpendicular magnetic anisotropy [5].

Here, we present scanning transmission electron microscopy (STEM) imaging and elemental analysis characterizing p-MTJ interfaces. The samples are thin films deposited onto oxidized silicon wafers by ultrahigh-vacuum magnetron sputtering. For sample preparation, crosssectional lamellae are prepared by focused-ion-beam milling. Thinning of the lamellae is critical to achieve electron-transparent specimens. Because p-MTJs are often sandwiched between transition metal elements with higher atomic numbers (higher $\mathrm{Z}$ values), the electron scattering in STEM due to the metal layers can be significant. The $\mathrm{CoFeB}$ and $\mathrm{MgO}$ layers contain relatively low-Z elements and are only $0.5-2 \mathrm{~nm}$ thick, compared to the high-Z metal layers that are $10-20 \mathrm{~nm}$ thick. Analytical STEM analysis, including electron energy loss spectroscopy (EELS) and energy dispersive X-ray spectroscopy (EDS), is uniquely suited to the compositional and morphological analysis of such fine features.

Figure 1 shows high-angle annular dark-field (HAADF) STEM images of a p-MTJ. In Figure $1 \mathrm{~A}$, the thin-film sample has been patterned and cross sectioned into a lamella for STEM analysis (from top to bottom: protective platinum and carbon layers, the p-MTJ structure, and Si regions of increasing thickness). Figure $1 \mathrm{~B}$ presents a higher-magnification STEM image of the p-MTJ structure (top: two metal layers; middle: the CoFeB/MgO/CoFeB p-MTJ; and bottom: three metal layers) [6]. 


\section{References}

[1] S Ikeda et al., Nat. Mater. 9 (2010) 721.

[2] WG Wang et al., Appl. Phys. Lett. 95 (2009) 242501.

[3] T Liu et al., Sci. Rep. 4 (2014) 5895.

[4] W-G Wang, et al., Nat. Mater. 11 (2012) 64.

[5] HD Gan et al., Appl. Phys. Lett. 99 (2011) 252507.

[6] The authors gratefully acknowledge the preparation of p-MTJ samples by the research group of Prof. Weigang Wang in the Department of Physics at the University of Arizona, and funding provided by the Center for Spintronics, a STARNET program administered by the Semiconductor Research Corporation.
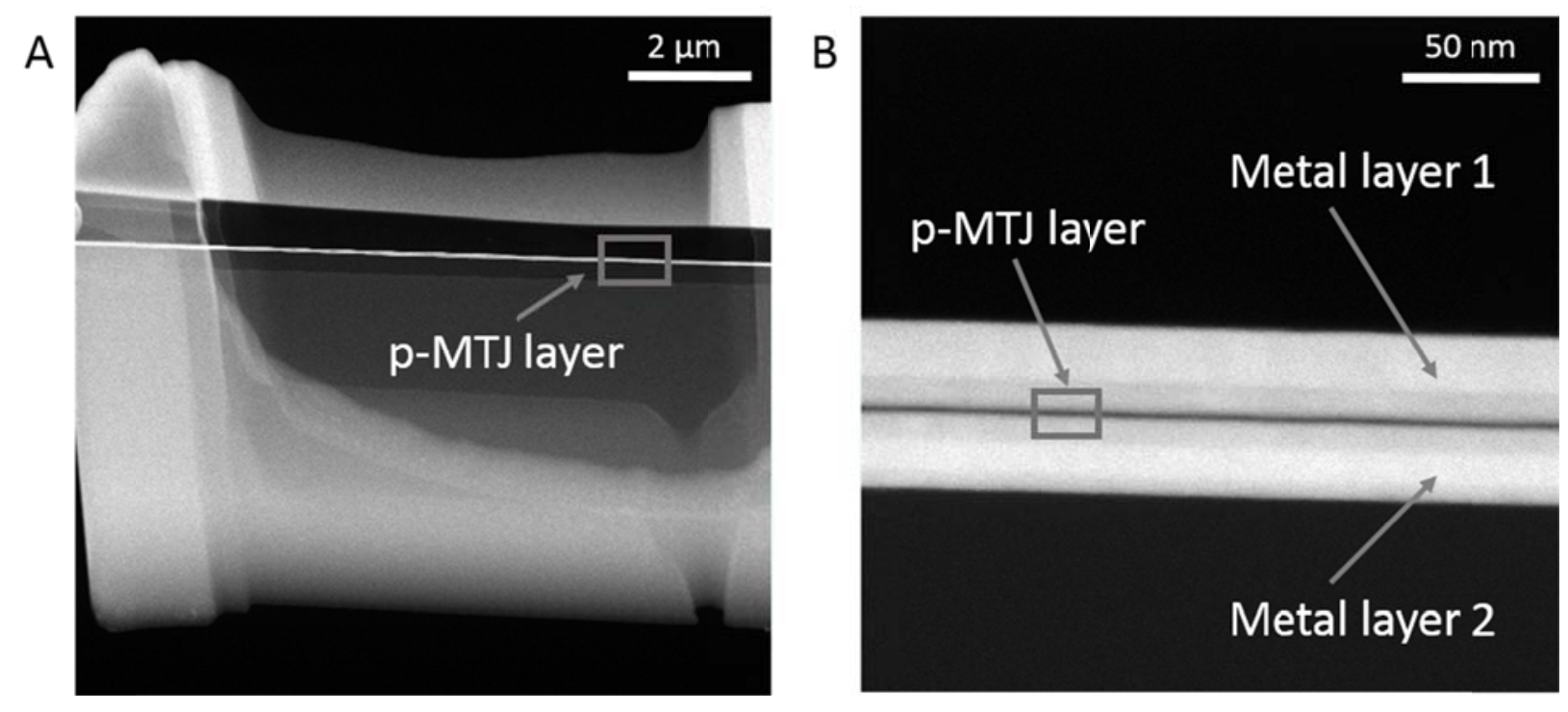

Figure 1. HAADF STEM images of p-MTJ samples: (A) image of the cross-sectional lamella and (B) image of the p-MTJ sandwiched between two multicomponent metal layers. 